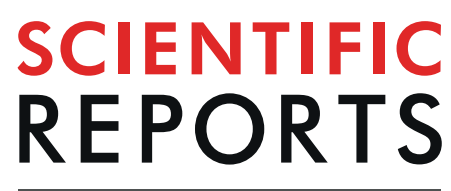

natureresearch

Check for updates

\title{
Destabilization of light NREM sleep by thalamic PLC $\beta 4$ deletion impairs sleep-dependent memory consolidation
}

\author{
Joohyeon Hong $\mathbb{1}^{1}$, Go Eun Ha ${ }^{1}$, Hankyul Kwak ${ }^{1}$, Yelin Lee $\mathbb{D}^{1}$, Hyeonyeong Jeong ${ }^{1}$, \\ Pann-Ghill Suh ${ }^{2,3}$ \& Eunji Cheong ${ }^{1 \otimes}$
}

Sleep abnormality often accompanies the impairment of cognitive function. Both rapid eye movement (REM) and non-REM (NREM) sleep have associated with improved memory performance. However, the role of composition in NREM sleep, consisting of light and deep NREM, for memory formation is not fully understood. We investigated how the dynamics of NREM sleep states influence memory consolidation. Thalamocortical (TC) neuron-specific phospholipase C 34 (PLC 34 ) knockout (KO) increased the total duration of NREM sleep, consisting of destabilized light NREM and stabilized deep NREM. Surprisingly, the longer NREM sleep did not improve memory consolidation but rather impaired it in TC-specific PLC $34 \mathrm{KO}$ mice. Memory function was positively correlated with the stability of light NREM and spindle activity occurring in maintained light NREM period. Our study suggests that a single molecule, PLC 34 , in TC neurons is critical for tuning the NREM sleep states and thus affects sleepdependent memory formation.

Sleep is a fundamental biological process that is well-preserved across most animals ${ }^{1}$. Sleep has crucial roles in brain metabolite clearance ${ }^{2}$, energy conservation ${ }^{3}$, brain plasticity ${ }^{4}$, and the process of cognition or emotions ${ }^{5}$. Many human studies suggest that the quantity and quality of sleep, such as duration, latency, architecture, and brain rhythms, affect memory function ${ }^{6-12}$. They report that a longer sleep duration supports memory consolidation $^{7}$, whereas sleep disruption is accompanied by memory deficits ${ }^{8,9}$. Impaired memory consolidation has been associated with shorter sleep duration and altered sleep-brain rhythms in several psychiatric disorders, such as major depression and schizophrenia ${ }^{10,11}$. Moreover, memory performance in psychiatric patients can be improved after sleep ${ }^{12}$. Thus, sleep is thought to benefit memory consolidation.

Sleep consists of non-rapid eye movement (NREM) and rapid eye movement (REM) sleep. In humans, NREM sleep is further subdivided into stages $1-4$ according to sleep depth ${ }^{13}$. NREM stages 1 and $2(\mathrm{~N} 1$ and N2) are classified as light NREM sleep. The N2 stage is characterized by the occurrence of a K-complex $(<1 \mathrm{~Hz})$ and a sleep spindle $(12-16 \mathrm{~Hz}$ in humans and $10-15 \mathrm{~Hz}$ in rodents) in electroencephalography (EEG) activity. N3 and N4 stages are classified as deep NREM sleep and are characterized by large-amplitude, low-frequency delta $(\delta)$ waves $(0.5-4 \mathrm{~Hz})$ in EEG activity. In most studies using rodent models, NREM sleep is not categorized into N1N4 stages. REM sleep is marked by distinctive regular theta $(\theta)$ waves. Several studies have investigated the effects of NREM versus REM sleep on memory formation to elucidate whether different sleep stages have distinct roles in various types of memories ${ }^{14,15}$. Human studies have suggested that deep NREM sleep predominantly benefits declarative memory consolidation (e.g., remembering events or facts), whereas REM sleep supports the consolidation of non-declarative memories (e.g., learning languages or movement-based skills) ${ }^{15,16}$. However, the role of light NREM sleep on memory consolidation is relatively unknown ${ }^{17}$.

During NREM sleep, large-amplitude oscillatory brain rhythms reflect the ensemble activities in thalamocortical circuit, which is composed of cortical, thalamocortical (TC) and thalamic reticular nuclei ${ }^{18}$. The thalamocortical circuit is thought to be the origin of sleep spindles and $\delta$ waves, which primarily occur in light and deep NREM sleep, respectively ${ }^{19,20}$. Among the many inputs to TC neurons, the corticothalamic pathway from cortical

${ }^{1}$ Department of Biotechnology, College of Life Science and Biotechnology, Yonsei University, Seoul, 03722, Republic of Korea. ${ }^{2}$ School of Life Science, Ulsan National Institute of Science and Technology, Ulsan, 44919, Republic of Korea. ${ }^{3}$ Korea Brain Research Institute, Daegu, 41062, Republic of Korea. ${ }^{\bowtie}$ e-mail: eunjicheong@yonsei.ac.kr 
neurons in layer VI sends massive glutamatergic inputs ${ }^{21}$. The activation of corticothalamic inputs has been reported to increase the excitability of TC neurons via the type 1 metabotropic glutamate receptor (mGluR1) ${ }^{22}$. mGluR1s are exclusively found in postsynaptic membranes that receive corticothalamic inputs, and are tightly linked to phospholipase C (PLC) $\beta 4$ in TC neurons ${ }^{23,24}$. Several studies report that PLC $\beta 4$ is highly expressed in TC neurons and is involved in regulating the firing properties of TC neurons, which affect thalamocortical oscillations ${ }^{25,26}$. In the present study, we investigated changes in light and deep NREM sleep and their effects on memory consolidation using TC-specific PLC $\beta 4$ knockout (KO) mice.

We found that TC-specific deletion of PLC 34 impaired sleep-dependent declarative memory consolidation, although the total duration of NREM sleep increased. Memory function was positively correlated with light NREM stability and spindle activity. The current results suggest that the thalamocortical circuit affects the dynamics of light and deep NREM sleep. Furthermore, light NREM sleep, and not just the total duration of NREM sleep, seem to be important for memory formation.

\section{Results}

TC-specific PLC $\beta 4$ KO decreased the duration of light NREM sleep. To examine the effect of the TC-specific PLC $\beta 4$ deletion on the natural sleep-wake pattern, AAV.eGFP (control) or AAV.eGFP-Cre (PLC 34 TC KO) were injected into the TC region of $P l_{c} \beta 4$ floxed mice (Fig. 1a). The mRNA expression levels of thalamic Plc $\beta 4$ were significantly lower in PLC $\beta 4$ TC KO mice than in control mice (Primer 1: control, $1.01 \pm 0.08$; PLCB4 TC KO, $0.35 \pm 0.07 ; \mathrm{p}=0.001$, unpaired t-tests; Primer 2: control, $1.02 \pm 0.11$; PLC $\beta 4$ TC KO, $0.31 \pm 0.05$; $\mathrm{p}=0.004$, unpaired t-tests; Fig. 1b,c). Additionally, we confirmed that AAV.eGFP was expressed in the cytosol and nuclei of TC neuronal cells (Fig. 1d). AAV.eGFP-Cre was specifically translocated to the nuclei of TC neurons and caused a significant reduction of PLC $\beta 4$ protein expression (AAV-eGFP, $95.96 \pm 1.34 \%$; AAV.eGFP-Cre, $4.26 \pm 4.26 \% ; \mathrm{p}=7 \times 10^{-5}$, unpaired t-tests; Fig. 1d,e). We further investigated the firing pattern of TC neurons labeled with GFP expression in control and PLC 34 TC KO mice (Fig. 1f) and found that the number of spike per burst was significantly increased in PLC $\beta 4$ KO TC neurons compared with control following various hyperpolarizing steps ( $-70 \mathrm{mV}$ : control, $0.6 \pm 0.6$; PLC $\beta 4 \mathrm{TC} \mathrm{KO,} 2.9 \pm 0.8 ; \mathrm{p}=0.04$, unpaired t-tests; $-80 \mathrm{mV}$ : control, $3.4 \pm 0.9 ; \mathrm{PLC} \beta 4 \mathrm{TC}$ KO, $6.0 \pm 0.5 ; \mathrm{p}=0.046$, unpaired t-tests; $-90 \mathrm{mV}$ : control, $4.4 \pm 0.9 ; \mathrm{PLC} \beta 4 \mathrm{TC}$ KO, $8.8 \pm 0.6$; $\mathrm{p}=0.007$, unpaired t-tests; Fig. $1 \mathrm{~g}$ ).

Both the control and PLC $\beta 4$ TC KO mice displayed typical and characteristic EEG and electromyography (EMG) patterns of wake and sleep states (Fig. 2a). The transition from wake to NREM sleep showed a reduction of EMG tone with high amplitudes and slow EEG patterns. NREM sleep was further subdivided into light (L.NR, ii and vi) and deep (D.NR, iii and vii) NREM states based on the ratio of $\delta$ band power ${ }^{27-30}$. Typically, low amplitude, regular EEG patterns in the $\theta$ frequency range were observed in REM sleep with EMG atonia (iv and viii). The hypnogram showing the change of vigilance state over $24 \mathrm{~h}$ revealed normal nocturnal activity with a diurnal sleep preference in both groups. It is noteworthy that NREM sleep mostly comprised light NREM in control mice, but mostly deep NREM in PLCB4 TC KO mice (Fig. 2b). In brain rhythms, the $\delta$ band power was enhanced in PLC 34 TC KO mice compared with control mice in NREM and REM sleep state during the light and dark phase (Light phase; NREM: control, $0.40 \pm 0.01$; PLCB4 TC KO, $0.47 \pm 0.009 ; \mathrm{p}=0.0007$, unpaired t-tests; REM: control, $0.14 \pm 0.005 ;$ PLC $\beta 4$ TC KO, $0.19 \pm 0.01 ; \mathrm{p}=0.0003$, unpaired t-tests; Dark phase; NREM: control, $0.46 \pm 0.01 ; \mathrm{PLC} \beta 4 \mathrm{TC} \mathrm{KO}, 0.51 \pm 0.01 ; \mathrm{p}=0.0003$, unpaired t-tests; REM: control, $0.16 \pm 0.006 ; \mathrm{PLC} \beta 4 \mathrm{TC} \mathrm{KO}$, $0.22 \pm 0.014 ; p=0.0015$, unpaired t-tests; Fig. 2c).

The total duration of NREM sleep significantly increased with a reduction of wake amount in PLC $\beta 4$ TC KO mice compared with control mice during the light phase (Wake: control, $217.63 \pm 5.96 \mathrm{~min}$; PLC $\beta 4$ TC $\mathrm{KO}, 176.34 \pm 7.85 \mathrm{~min} ; \mathrm{p}=0.0005$, unpaired t-tests; NREM: control, $453.01 \pm 6.37 \mathrm{~min}$; PLC $\beta 4 \mathrm{TC}$ KO, $499.844 \pm 7.91 \mathrm{~min} ; \mathrm{p}=0.0002$, unpaired t-tests; Fig. 2d). However, the total duration of REM sleep was unchanged in PLC 34 TC KO mice compared with control mice (Fig. 2d). In the dark phase, there were no differences in the total duration of wake and sleep states between the two groups (Fig. 2d). In NREM sleep, the total duration of light NREM significantly decreased, whereas the duration of deep NREM was elevated in PLC34 TC KO mice compared with control mice during the light phase (L.NR; control, $304.03 \pm 23.12 \mathrm{~min}$; PLCB4 TC KO, $203.92 \pm 20.04$ min; $\mathrm{p}=0.004$, unpaired t-tests; D.NR; control, $148.99 \pm 25.56 \mathrm{~min}$; PLC $\beta 4$ TC KO, $295.92 \pm 21.80 \mathrm{~min} ; \mathrm{p}=0.0003$, unpaired t-tests; Fig. 2e). It is noteworthy that NREM sleep consisted of a greater percentage of light NREM than the deep NREM in control mice, but this pattern was reversed in PLC $\beta 4$ TC KO mice, which exhibited much longer deep NREM sleep (L.NR; control, 67.41 \pm 5.42\%; PLC $\beta 4$ TC KO, $40.90 \pm 4.13 \% ; \mathrm{p}=0.001$, unpaired t-tests; D.NR; control, $32.59 \pm 5.42 \%$; PLC 34 TC KO, 59.10 $\pm 4.13 \%$; $\mathrm{p}=0.001$, unpaired t-tests; Fig. 2f). These results suggest that TC-specific PLC $\beta 4$ deletion decreased the duration of light NREM sleep, yet increased the total duration of NREM sleep.

Each episode of light NREM sleep was destabilized whereas episodes of deep NREM sleep were more frequent and longer in TC-specific PLC $34 \mathrm{KO}$ mice. To distinguish if the altered durations of light and deep NREM sleep were due to a change in either the occurrence or the maintenance of each episode, we analyzed the number and duration of episodes in each state. PLC $\beta 4$ TC KO mice showed a greater number of deep NREM episodes (control, $104.50 \pm 8.41$; PLCB4 TC KO, $135.67 \pm 6.15 ; \mathrm{p}=0.008$, unpaired t-tests; Fig. 3a) and a significantly longer episodic duration of deep NREM (control, $80.40 \pm 9.04$ sec; PLC 34 TC KO, $132.50 \pm 11.62 \mathrm{sec} ; \mathrm{p}=0.002$, unpaired t-tests; Fig. $3 \mathrm{~b}$ ). There was no significant difference in the number of light NREM episodes between groups (Fig. 3a), but PLC 34 TC KO mice showed a significantly shorter episodic duration of light NREM (control, $129.20 \pm 13.60 \mathrm{sec}$; PLC 34 TC KO, 80.25 $\pm 6.56 \mathrm{sec} ; \mathrm{p}=0.006$, unpaired t-tests; Fig. $3 \mathrm{~b}$ ). These results indicate that the total duration of deep NREM was increased by the enhancement of both occurrence and maintenance in PLC 34 TC KO. However, the decrease in the total duration of light NREM was caused by weakened maintenance and not by occurrence. 
a
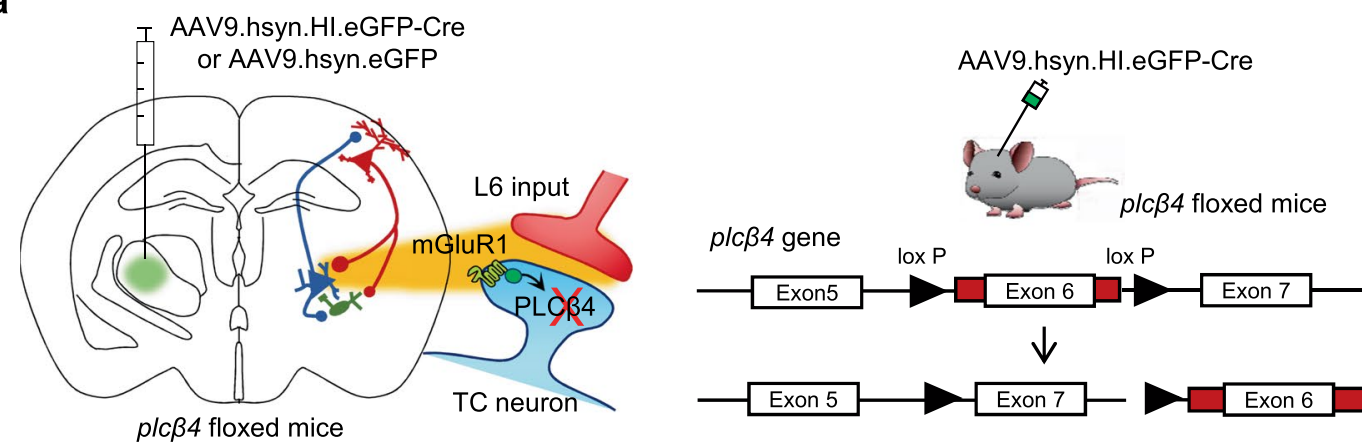

b

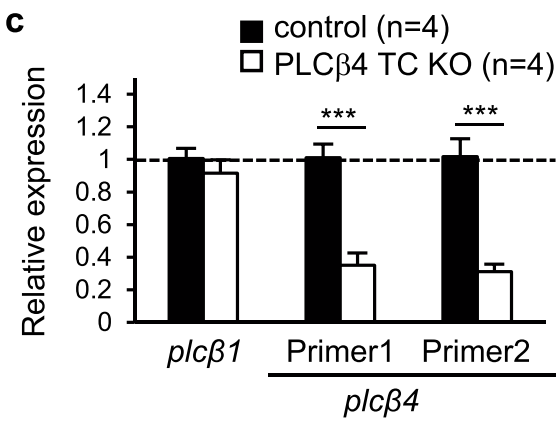

d

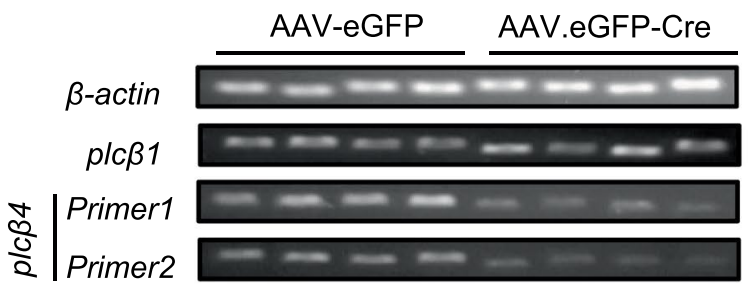

e
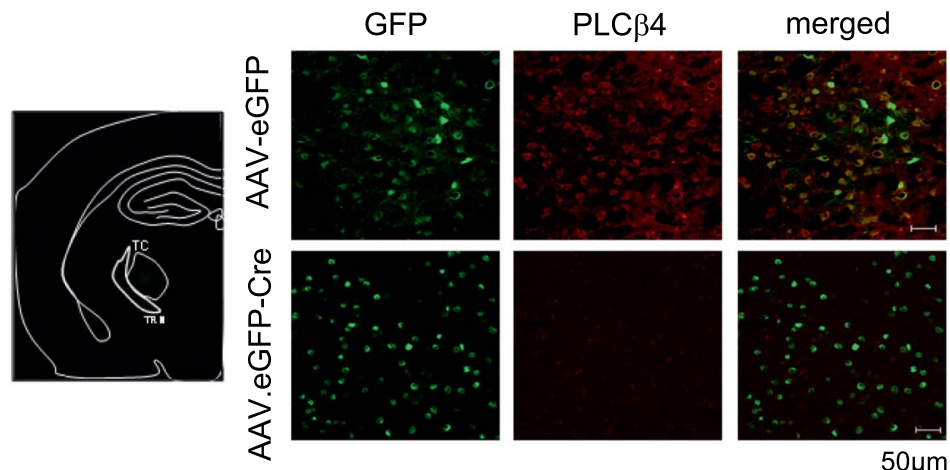

믄이 $(n=4)$
PLC $\beta 4$ TC KO $(n=4)$

f

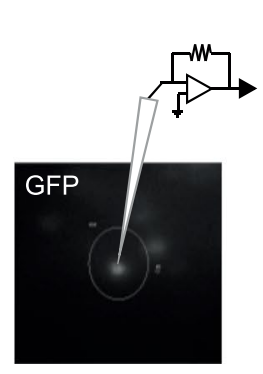

AAV-eGFP

$20 \mathrm{mV}$

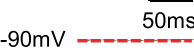

AAV.eGFP-Cre

$-90 \mathrm{mV}$

ms

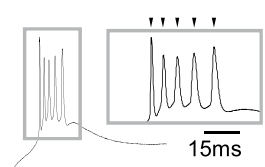

r
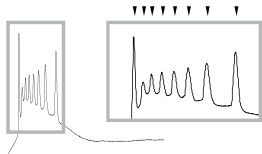

g

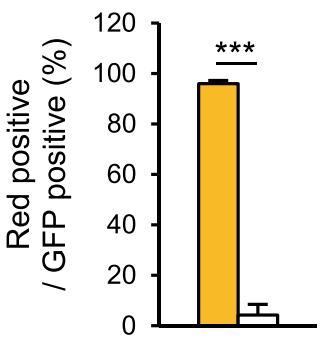

$50 \mu \mathrm{m}$

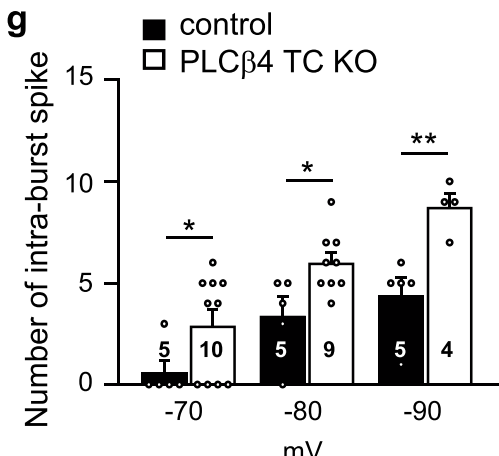

Figure 1. The deletion of PLC $\beta 4$ in thalamic neurons of $p l c \beta 4$ floxed mice. (a) Schematic showing bilateral injection of AAV9.hsyn.HI.eGFP-Cre.WPRE. SV40 (PLC34 TC KO) or AAV9. hsyn.eGFP.WPRE.Bgh

(control) in the TC regions of Plc $\beta 4$ floxed transgenic mice. Exons 6 in alleles of the Plc $\beta 4$ gene was deleted by Cre recombinase (right panel). (b) RT-PCR data assessing Plc $\beta 4$ expression for confirmation of the Cre-loxP system. The grouping of gels were cropped from different gels by genes ( $P l c \beta 1, P l c \beta 4$ (Primer 1,2$)$ and $\beta$-actin). Uncropped gels are shown in Supplementary Fig. S1. (c) The quantitative mRNA expression levels of $P l c \beta 1$ and Plc $\beta 4$ normalized to $\beta$-actin (control, $\mathrm{n}=4$, black bar; PLC $\beta 4$ TC KO, $\mathrm{n}=4$, white bar). (d) Representative fluorescence image of viral expression (green) in the TC regions with PLC $\beta 4$ staining (red). Scale bar represents $50 \mu \mathrm{m}$. (e) The quantitative PLC $\beta 4$ expression was reduced in TC neurons of PLC $\beta 4$ TC KO mice (control, $\mathrm{n}=4$, yellow bar; PLC $\beta 4$ TC KO, $\mathrm{n}=4$, white bar). (f) Representative traces of rebound burst firing recorded from TC neurons in ventrobasal complex region infected with AAV-eGFP or AAV-eGFP-Cre under currentclamp configuration. (g) The number of intra-burst spikes at $-70,-80$, or $-90 \mathrm{mV}$. (control, $\mathrm{n}=5$, black bar; PLC34 TC KO, $-70 \mathrm{mV} ; \mathrm{n}=10,-80 \mathrm{mV} ; \mathrm{n}=9,-90 \mathrm{mV} ; \mathrm{n}=4$, white bar). Data are represented as the mean \pm standard error of the mean (SEM). ${ }^{*} \mathrm{p}<0.05 ;{ }^{* *} \mathrm{p}<0.01 ;{ }^{* * *} \mathrm{p}<0.005$. 
a AAV9.hsyn.HI.eGFP-Cre AAV9.hsyn.eGFP

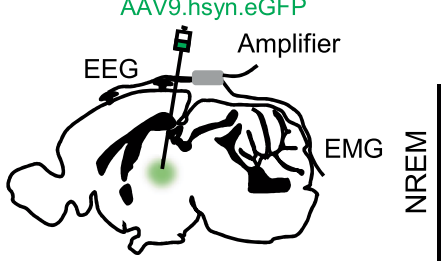

plc $\beta 4$ floxed mice

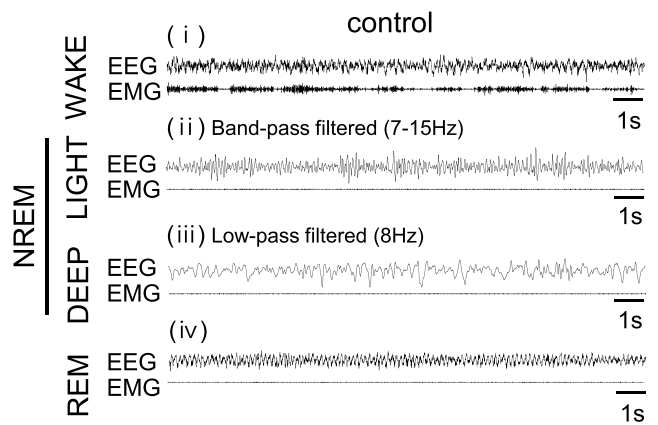

- W: Wake

- L.NR : Light NREM sleep - D.NR : Deep NREM sleep

b

- R : REM sleep
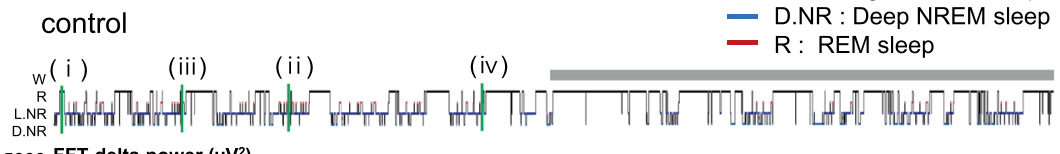
5000 FFT delta power $\left(\mu \mathrm{V}^{2}\right)$

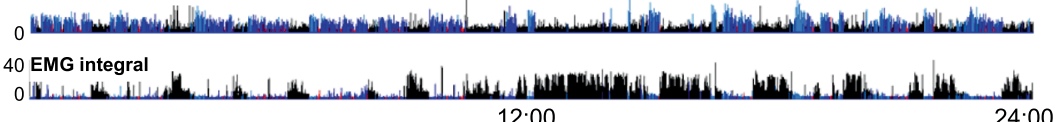

PLC $\beta 4$ TC KO

$w^{\prime}(\mathrm{v}) \quad$ (vi) (vii) (viii)

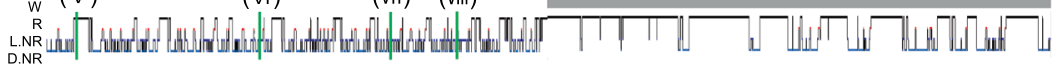
7000 FFT delta power $\left(\mu \mathrm{V}^{2}\right)$

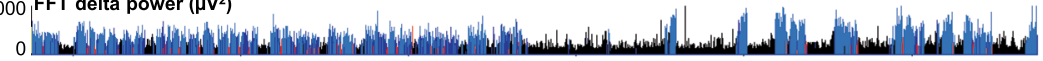
40 EMG integral

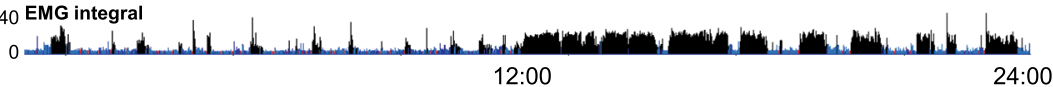

$12: 00$

d

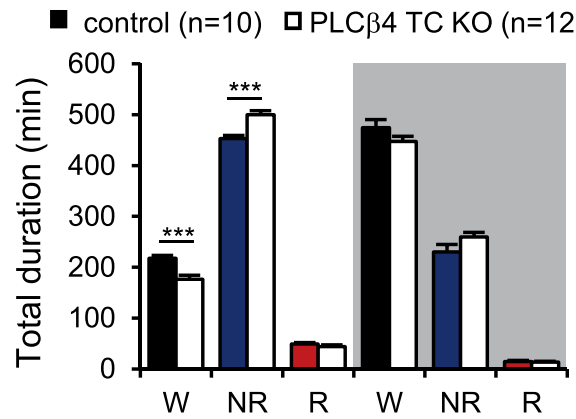

e

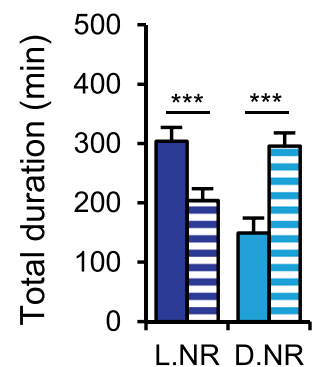

(v) PLC 34 TC KO

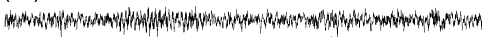

(vi)

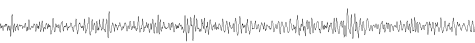

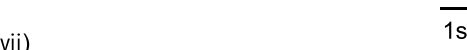

(vii)

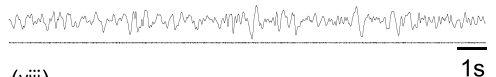

(viii) $\overline{1 \mathrm{~s}}$

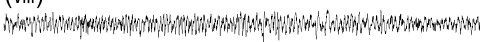$$
\text { C }
$$

\section{C}

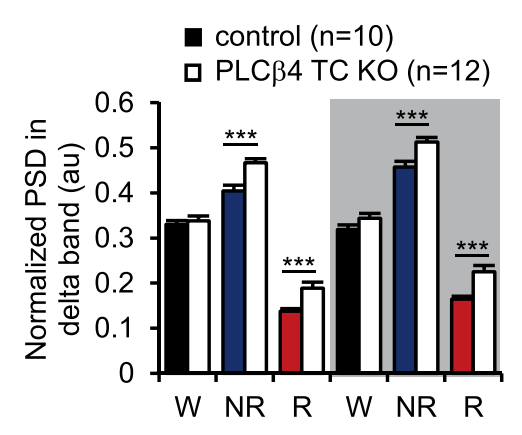

Figure 2. The reversed composition of light and deep NREM sleep by TC-specific PLCB4 deletion. (a) Representative EEG and EMG traces during awake, light NREM, deep NREM, and REM sleep. The position of each trace (i-viii) was indicated in the hypnogram. (b) Representative hypnograms with fast Fourier transformation-derived $\delta$ band power and integrated EMG activity over $24 \mathrm{~h}$. The y-axis of the hypnograms shows the state of vigilance, and the $\mathrm{x}$-axis shows the $24 \mathrm{~h}$ period that included one light and one dark (gray bars) cycle. (c) Normalized power spectrum density (PSD) for the $\delta$ bands (arbitrary units, au) in awake (W), NREM (NR), and REM (R) sleep during the light and dark phases. (d) The total time spent in W, NR, and R sleep during the light and dark phases. (e) The total time spent in the light NREM (L.NR) and deep NREM (D.NR) sleep during the light phase. (f) The average percentage of L.NR and D.NR in total NREM sleep in the control and PLC $\beta 4$ TC KO mice (control, $\mathrm{n}=10$, colored bar; PLC $\beta 4$ TC KO, $\mathrm{n}=12$, white or graduated bar). Data are represented as the mean \pm standard error of the mean (SEM) ${ }^{* * * *} \mathrm{p}<0.005$.

Next, we analyzed the transition probability from one state to other states. In control mice, the transition from awake to NREM sleep was dominated by a transition from awake to light NREM (W-L.NR, 65\%, Fig. 3c), which is considered normal in human sleep ${ }^{31}$. However, in the PLC 34 TC KO mice, the W-L.NR transition was reduced to $\sim 45 \%$, whereas W-D.NR transition increased by almost 55\% (W-L.NR: control, $65.31 \pm 3.26 \%$; PLC 34 TC KO, $45.03 \pm 5.39 \% ; \mathrm{p}=0.0049$, unpaired t-tests; W-D.NR: control, $34.69 \pm 3.26 \%$; PLC $\beta 4$ TC KO, $54.97 \pm 5.39 \%$; $\mathrm{p}=0.0049$, unpaired t-tests; Fig. 3c). Once the light NREM sleep was initiated in PLCB4 TC KO mice, it transitioned more frequently into deep NREM sleep than it did in control mice (L.NR-D.NR: control, $58.05 \pm 3.14 \%$; PLCB4 TC KO, 66.42 $\pm 1.67 \%$; $=0.03$, unpaired t-tests; Fig. 3c). The L.NR to REM (L.NR-R) transition was less frequent in PLC $\beta 4$ TC KO mice than control. Instead, the D.NR-R transition, which is abnormal sleep progress 
a

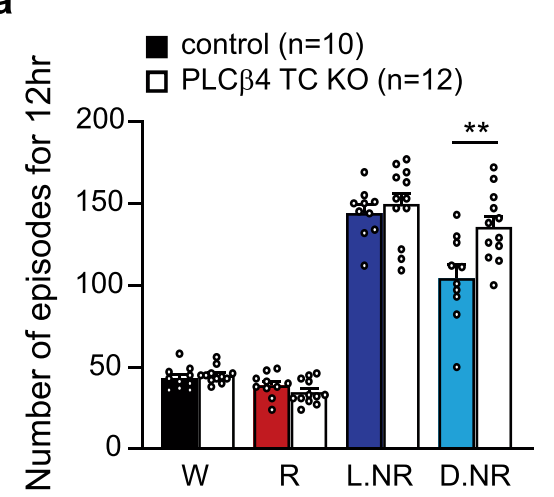

b

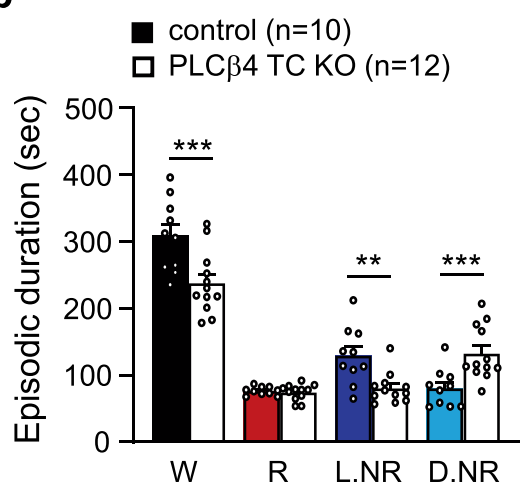

C

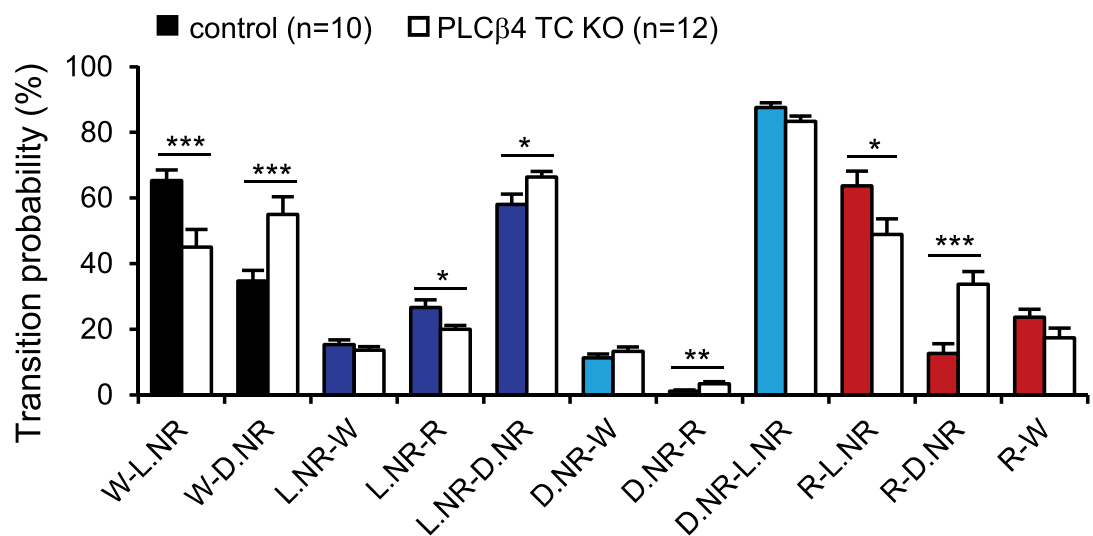

d

In control (normal condition)

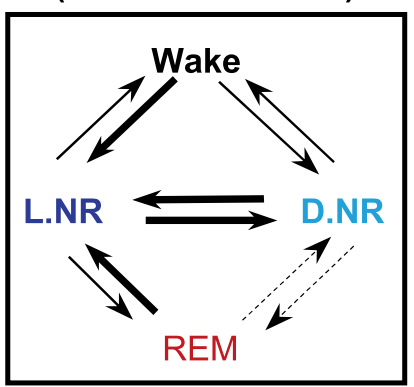

In PLC $\beta 4$ TC KO

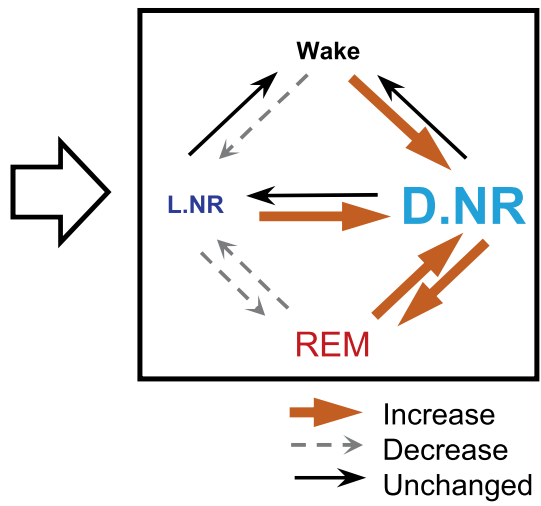

Figure 3. Altered dynamics in light and deep NREM sleep by TC-specific PLC $\beta 4$ deletion. (a) The number of episodes of each vigilance state during the light phase. (b) The episodic duration of each vigilance state during the light phase. (c) The transition probability between wake and sleep states. (d) The scheme of transition probabilities among wake and sleep states in control and PLC 34 TC KO mice. Thick (orange) and dotted (gray) arrows indicate increase and decrease of the transition tendency in PLC 34 TC KO mice compared with control mice. The data were obtained from control ( $\mathrm{n}=10$, colored bar) and PLC 34 TC KO mice ( $\mathrm{n}=12$, white bar). Data are represented as the mean \pm standard error of the mean (SEM). ${ }^{*} \mathrm{p}<0.05 ;{ }^{* *} \mathrm{p}<0.01 ;{ }^{* * *} \mathrm{p}<0.005$

rarely observed in controls, was often occurred in PLC $\beta 4$ TC KO mice (L.NR-R: control, $26.63 \pm 2.35 \%$; PLC $\beta 4$ TC KO, $20.00 \pm 1.16 \%$; $\mathrm{p}=0.02$, unpaired t-tests; D.NR-R: control, $1.14 \pm 0.32 \%$; PLC $\beta 4$ TC KO, $3.40 \pm 0.62 \%$; $\mathrm{p}=0.005$, unpaired t-tests; Fig. 3c). From REM sleep, the R-L.NR transition was reduced, whereas R-D.NR transition increased in PLC $\beta 4$ TC KO mice than in control mice (R-L.NR: control, $63.74 \pm 4.42 \%$; PLC $\beta 4$ TC $\mathrm{KO}, 48.93 \pm 4.76 \%$; $\mathrm{p}=0.03$, unpaired t-tests; R-D.NR: control, $12.61 \pm 2.99 \%$; PLC $\beta 4$ TC KO, $33.73 \pm 3.85 \%$; $\mathrm{p}=0.0003$, unpaired t-tests; Fig. 3c). Altogether, these results showed that the relative transition to deep NREM sleep significantly increased in PLC 34 TC KO mice, which resulted in a stabilized deep NREM sleep and destabilized light NREM sleep (Fig. 3d). These results indicate that changes restricted to TC neurons dramatically 
a

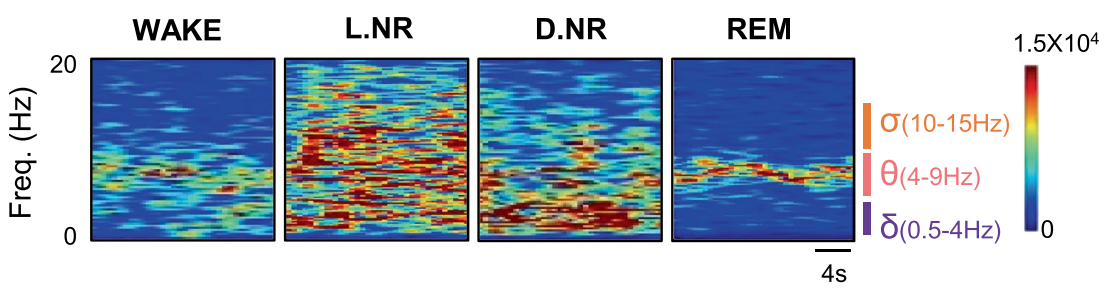

C

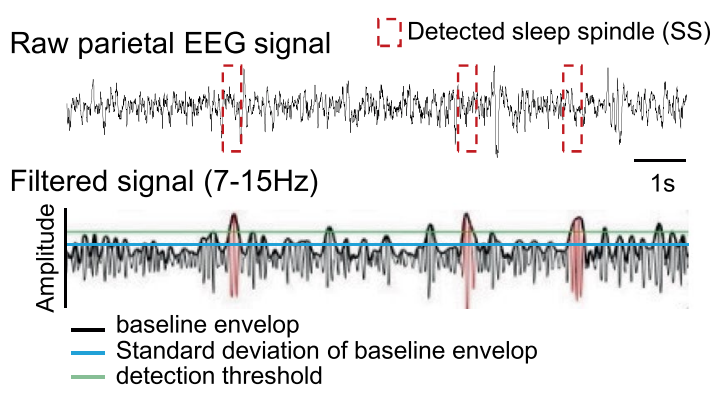

b

control $(n=10)$

PLC $\beta 4$ TC KO $(n=12)$

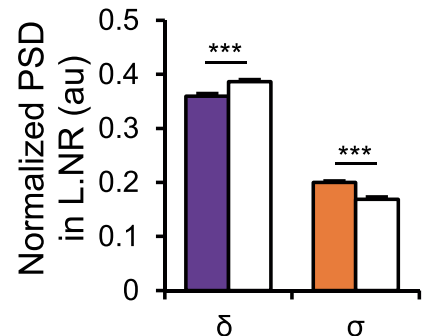

e

d

a control $(n=9)$

口 PLC $\beta 4$ TC KO $(n=11)$

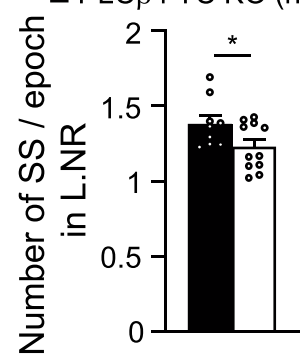

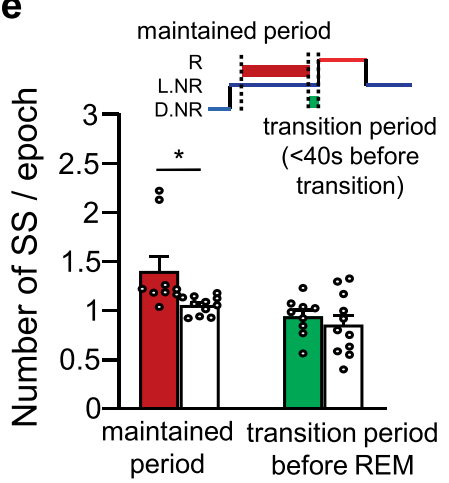

f

control $(n=10)$

口 PLC 34 TC KO $(n=12)$

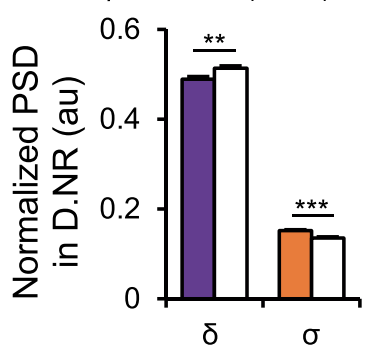

g

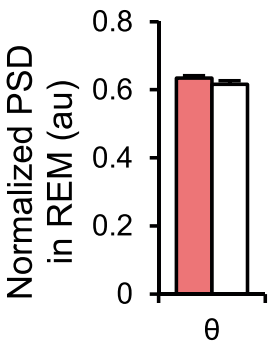

h

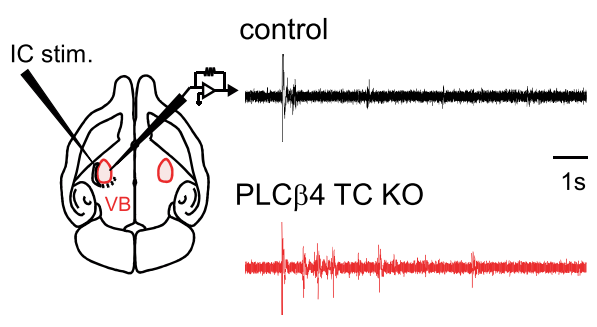

i

control $(n=5)$ 口 PLC $\beta 4$ TC KO $(n=7)$

Figure 4. The contrast changes in brain rhythms of light and deep NREM sleep by the TC-specific PLC $\beta 4$ deletion. (a) Representative spectrograms in wake and sleep states. (b) Normalized power spectrum density (PSD) for the $\delta(0.5-4 \mathrm{~Hz})$ and $\sigma(10-15 \mathrm{~Hz})$ bands (arbitrary units, au) in the light NREM sleep (L.NR). (c) Representative traces illustrating the spindle detection protocol. (d) The number of sleep spindle (SS) per epoch occurred in the L.NR state was significantly reduced in the PLC 34 TC KO mice. (e) The number of SS per epoch occurred in the L.NR state, which was maintained (red) or right before transited to the REM sleep (green) (control, $\mathrm{n}=9$, colored bar; PLC $\beta 4$ TC KO, $\mathrm{n}=11$, white bar). (f) Normalized PSD for the $\delta$ and $\sigma$ bands in the deep NREM sleep (D.NR). (g) Normalized PSD for the $\theta(4-9 \mathrm{~Hz})$ band in REM sleep. All PSD data were obtained from control ( $\mathrm{n}=10$, colored bar) and PLC $\beta 4$ TC KO mice ( $\mathrm{n}=12$, white bar). (h) Schematic illustration of intra-thalamic oscillation recording in the ventro-posterolateral nuclei (left). Representative traces of thalamic oscillations in control and PLC 34 TC KO mice (right). (i) The duration of intra-thalamic oscillatory activity increased in PLC $\beta 4$ TC KO mice (control, $\mathrm{n}=5$, black bar; PLC $\beta 4$ TC KO, $\mathrm{n}=7$, white bar). Data are represented as the mean \pm standard error of the mean $(\mathrm{SEM}) .{ }^{*} \mathrm{p}<0.05 ;{ }^{* *} \mathrm{p}<0.01 ;{ }^{* * *} \mathrm{p}<0.005$.

altered the NREM sleep composition, which is subdivided based on sleep depth. This led us to further investigate sleep-brain rhythms known to be generated in thalamocortical circuit during NREM sleep.

Spindle activity in light NREM sleep decreased in TC-specific PLC 34 KO mice. Next, we analyzed the power of brain rhythms representing each sleep state, independent of the duration of state (Fig. 4a). In the light NREM state, the sigma $(\sigma)$ band power significantly decreased with the enhancement of the $\delta$ band power in PLC 34 TC KO mice compared with control mice ( $\sigma$ : control, $0.20 \pm 0.003$; PLC 34 TC KO, $0.17 \pm 0.004$; $\mathrm{p}=5.85 \times 10^{-6}$, unpaired t-tests; $\delta$ : control, $0.36 \pm 0.005 ;$ PLC $\beta 4$ TC KO, $0.39 \pm 0.004 ; \mathrm{p}=0.0002$, unpaired t-tests; Fig. 4b). Next, we counted and analyzed the spindle activity as the average number of spindles per 1 epoch $(8 \mathrm{sec})$ in the light NREM state during which spindles are most commonly observed (Fig. $4 \mathrm{c})$. The number of spindle per epoch decreased in PLC $\beta 4$ TC KO mice compared to control mice (control, $1.38 \pm 0.05$; PLC $\beta 4$ 
TC KO, $1.23 \pm 0.05 ; \mathrm{p}=0.049$, unpaired t-tests; Fig. $4 \mathrm{~d}$ ). The total number of spindles in the light NREM state also decreased in PLC 34 TC KO mice (Supplementary Fig. S2a). In the deep NREM state, there was no significant difference in the number of spindle per epoch between groups, but the total number of spindles increased in PLC 34 TC KO mice compared to control mice (Supplementary Fig. S2a and S2b). It has been reported that spindle occurrence increases immediately before NREM to REM transition ${ }^{32}$. We therefore investigated if the number of spindle events in the light NREM to REM transition period ( $<40 \mathrm{sec}$ before transition occurrence) differed between groups, since both the number of spindle and the L.NR to R transition decreased in PLC 34 TC $\mathrm{KO}$ mice (Figs. 3d and 4d). The number of spindle per epoch in the transition period was not changed in PLCB4 TC KO mice compared to control mice. However, the spindle occurring in the maintained light NREM period, excluding the transition period, significantly decreased in PLC $\beta 4$ TC KO mice (control, $1.40 \pm 0.15$; PLC $\beta 4$ TC $\mathrm{KO}, 1.06 \pm 0.03 ; \mathrm{p}=0.047$, unpaired t-tests; Fig. 4e). These results indicate that TC-specific PLC $\beta 4$ KO decreased the spindle occurrence in the maintained light NREM period.

In the deep NREM state, $\delta$ band power significantly increased, with the reduction of the $\sigma$ band power in PLCß4 TC KO mice compared with control mice ( $\delta$ : control, $0.49 \pm 0.005 ;$ PLC $\beta 4$ TC KO, $0.51 \pm 0.005 ; \mathrm{p}=0.006$, unpaired t-tests; $\sigma$ : control, $0.15 \pm 0.002$; PLC $\beta 4$ TC KO, $0.14 \pm 0.003 ; \mathrm{p}=9.1 \times 10^{-5}$, unpaired t-tests; Fig. $\left.4 \mathrm{f}\right)$. In the REM state, there was no change in $\theta$ band power between groups (Fig. $4 \mathrm{~g}$ ). The slow intra-thalamic oscillatory activity $^{33}$, which was recorded in horizontal thalamocortical slices in vitro, was enhanced in PLC 34 TC KO mice (control, $2.26 \pm 0.77 \mathrm{sec}$; PLC $\beta 4$ TC KO, $4.61 \pm 0.61 \mathrm{sec} ; \mathrm{p}=0.042$, unpaired t-tests; Fig. 4h,i), which was parallel with increased $\delta$ power in NREM sleep. These results indicate that spindle activity and $\delta$ band power were regulated via a distinct mechanism, although both brain rhythms were generated in thalamocortical circuit.

Declarative memory consolidation was impaired in TC-specific PLC $\beta 4$ KO mice, regardless of longer NREM sleep. Longer NREM sleep is reported to benefit memory formation ${ }^{14}$. We investigated the effect of increased NREM sleep duration in TC-specific PLC34 KO mice on sleep-dependent memory consolidation. First, a novel object recognition task was performed to test declarative memory (Fig. 5a). Strikingly, the discrimination index (DI) was significantly lower in PLC $\beta 4$ TC KO mice than in control mice (day 2: control, $0.37 \pm 0.16$; PLC $\beta 4$ TC KO, $-0.08 \pm 0.06 ; \mathrm{p}=0.028$, unpaired t-tests; Fig. $5 b$ ), which indicates that memory consolidation was impaired. During the task, the difference between two groups was not observed in the approach number to objects (except for new object) or total distance moved (Fig. 5c,d). These showed that PLC 34 TC KO mice has normal sensory and cognitive functions like the control mice. To examine non-declarative memory function, the same mice completed a fear conditioning task and both control and PLC 34 TC KO mice showed normal learning function in the conditioning session (Fig. 5e,f). There were no between-group differences in the context- and cue-recall memory consolidations (Fig. 5g,h).

Next, sleep-independent memory function was tested using a Y-maze task, in which sleep was not permitted between the training and test sessions. There was no change in the preference for a novel arm between groups, which indicated that sleep-independent memory consolidation was normal in PLC $\beta 4$ TC KO mice (Fig. 5i). Both groups showed general locomotor activities with normal anxiety levels in the open field test (Fig. 5j). These results indicate that the TC-specific PLC $\beta 4$ deletion impaired sleep-dependent declarative memory consolidation, despite the increase in NREM sleep duration. These results prompted us to further investigate which detailed sleep components, such as stability of each episode, transition pattern between vigilance states and brain rhythms, rather than just the total NREM sleep duration, were related to memory deficits in PLCB4 TC KO mice.

Memory consolidation was positively correlated with light NREM stability. TC-specific PLC $\beta 4$ KO mice were characterized by a destabilized light NREM sleep, but with a stabilized deep NREM sleep (Fig. 3d). We therefore performed regression analyses between the degree of memory consolidation and components in the light and deep NREM sleep in all the mice tested. The degree of memory consolidation was expressed as a DI for a novel object. Among the many components of light NREM sleep, the episodic duration, indicating stability, showed a significant positive correlation with memory consolidation $(\mathrm{r}=0.55 ; \mathrm{p}=0.018$; Fig. 6a). However, there was no correlation between the episodic duration of deep NREM sleep and memory consolidation. $(r=-0.37$; $\mathrm{p}=0.13$; Fig. $6 \mathrm{~b}$ ). Interestingly, the total amount of NREM for $12 \mathrm{hr}$, especially deep NREM sleep, had negative correlation with memory consolidation (NR: $r=-0.48 ; p=0.04$; D.NR: $r=-0.48 ; p=0.04$; Supplementary Fig. S3a and S3b). These results suggest that light NREM sleep tightly linked to the consolidation of declarative memory via its stability, irrespective of the total amount of NREM sleep (Fig. 6g).

We then determined whether the decreased spindle activity in light NREM (Fig. 4d) or enhanced $\delta$ power in deep NREM (Fig. 4f) contributed more to the memory deficit in PLC $\beta 4$ TC KO mice. There was a strong positive correlation between the number of spindle per epoch occurring in the light NREM sleep and memory consolidation $(r=0.59 ; p=0.009$; Fig. $6 c)$. However, $\delta$ power of the deep NREM sleep did not correlate with memory consolidation $(\mathrm{r}=-0.38 ; \mathrm{p}=0.12$, Fig. $6 \mathrm{~d}$ ). Overall, these results indicate that spindle activity during light NREM sleep has a positive effect on declarative memory consolidation (Fig. $6 \mathrm{~g}$ ).

Sleep spindles occur throughout NREM sleep and are not limited to light NREM sleep. However, it is not known whether the role of spindles in memory functions is temporal-dependent. To investigate this, we divided the spindle into those occurring in maintained light or deep NREM periods and those occurring in the transition period from light to deep NREM sleep. A strong correlation with memory consolidation was shown only when the spindle occurred in maintained light NREM period $(r=0.70 ; p=0.0011$; Fig. 6e). There was no correlation between the number of spindle per epoch in maintained deep NREM period and memory consolidation $(r=-0.18 ; p=0.48$; Fig. 6f). Furthermore, the number of spindle per epoch in the transition period from light to deep NREM did not correlate with memory consolidation $(r=-0.04 ; p=0.88$; Supplementary Fig. S3c). These results indicate that the positive effect of spindle activity on declarative memory consolidation was specific for spindles that occurred during the maintained light NREM period (Fig. 6g). 


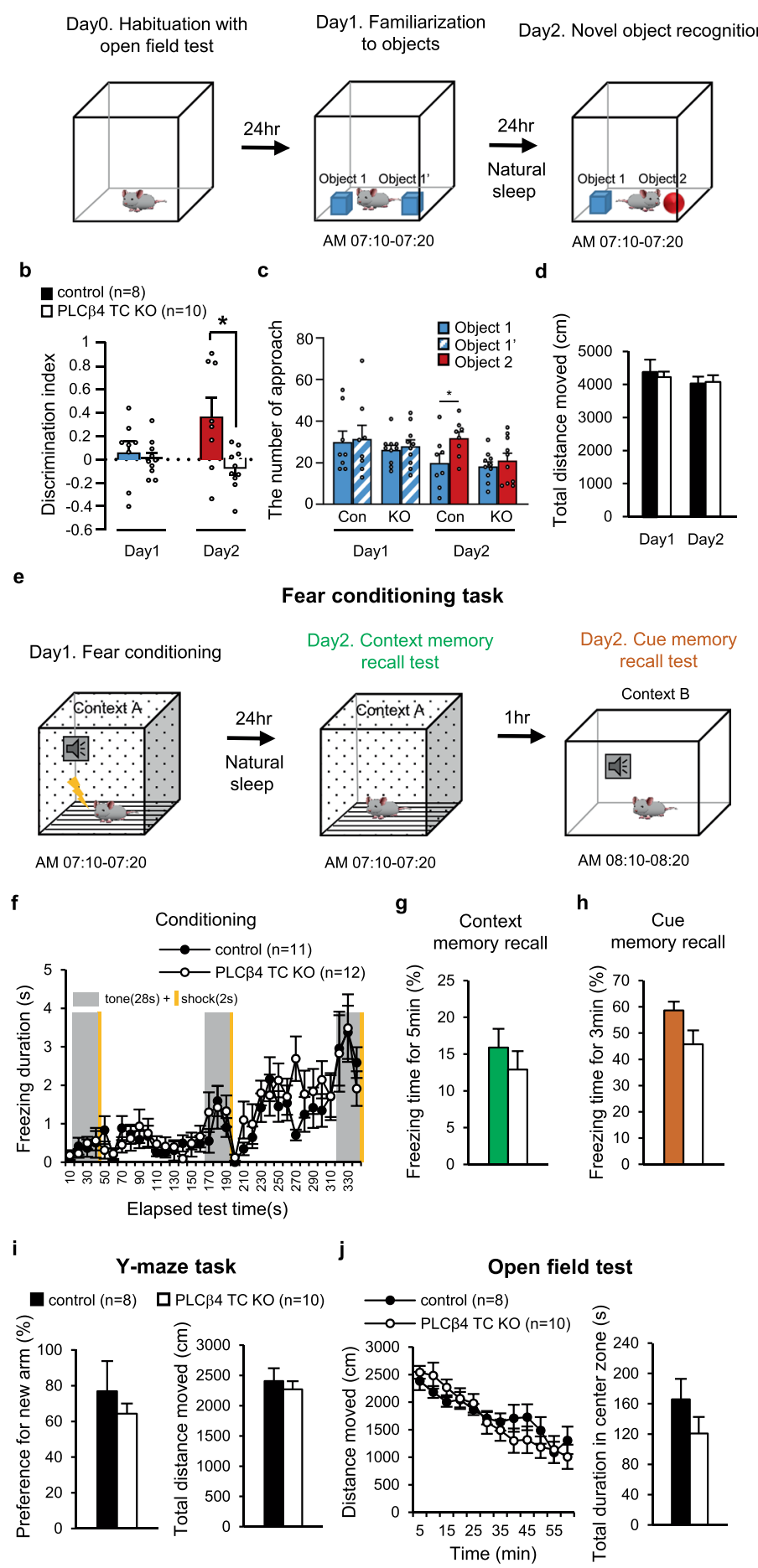

Figure 5. Sleep-dependent declarative memory impairment by TC-specific PLCB4 deletion. (a) A schematic illustration of the novel object recognition task. (b) The discrimination index during the training (day 1) and test session (day 2) (control, $\mathrm{n}=8$, colored bar; PLC $\beta 4$ TC KO, $\mathrm{n}=10$, white bar). (c) The number of approach for each object during the training and test session (control, $n=8$; PLCB4 TC KO, $n=10$; blue bar, object 1; blue grid bar, object 1', red bar, object 2). (d) The total distance moved for 10 min during the training and test session (control, $\mathrm{n}=8$, black bar; PLC $\beta 4$ TC KO, $\mathrm{n}=10$, white bar). (e) A schematic illustration of the fear conditioning task. (f) Time course of the freezing behavior during the conditioning phase. The conditioning session consisted of a 28 -sec tone (gray box) followed by a 2 -sec shock (yellow line) delivered at 2 -min intervals. Each point represents a 10-sec bin. (g,h) Freezing time during the context- and cue-memory recall tests. (control, $\mathrm{n}=11$, colored bar; PLC $\beta 4$ TC KO, $\mathrm{n}=12$, white bar). (i) Y-maze task for testing the sleep-independent memory function. (control, $\mathrm{n}=8$, black bar; PLC $\beta 4 \mathrm{TC} K \mathrm{KO}, \mathrm{n}=10$, white bar). (j) Open field test for testing locomotor activity (left) with the anxiety level (right) (control, $\mathrm{n}=8$, black bar; PLC $\beta 4$ TC KO, $\mathrm{n}=10$, white bar). Data are represented as the mean \pm standard error of the mean (SEM). ${ }^{*} \mathrm{p}<0.05$. 
a

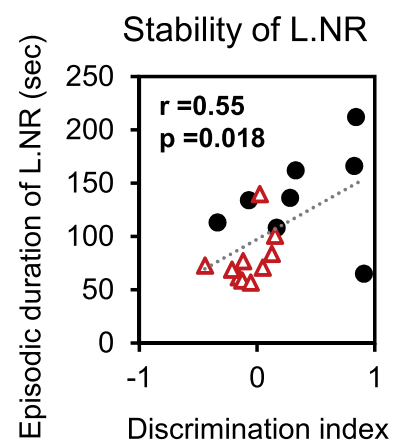

e
Spindle activity
in maintained L.NR

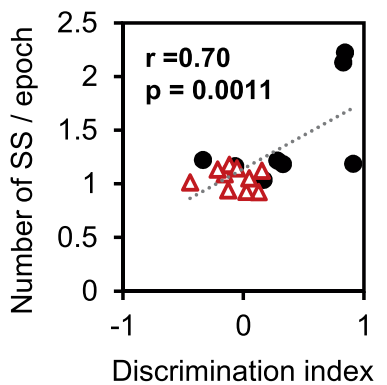

b

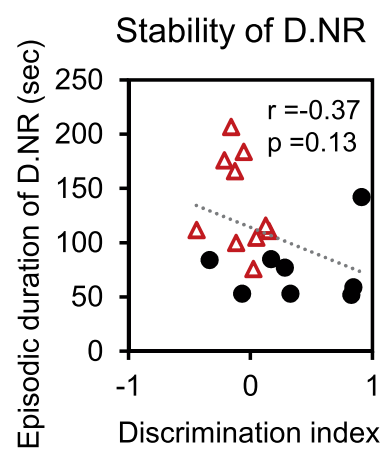

C

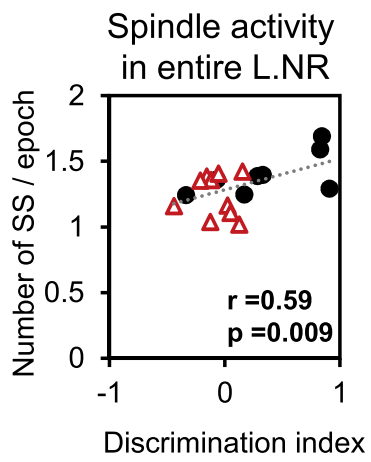

d

Delta power in D.NR

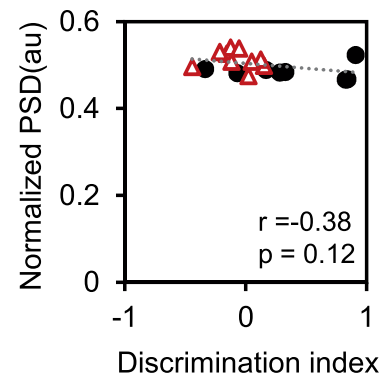

f

Spindle activity in maintained D.NR

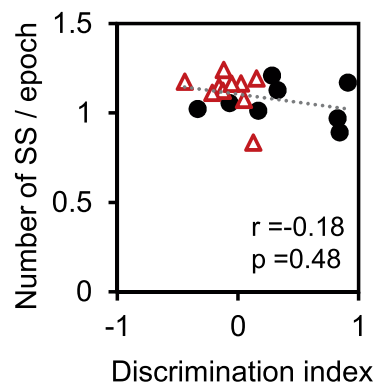

g

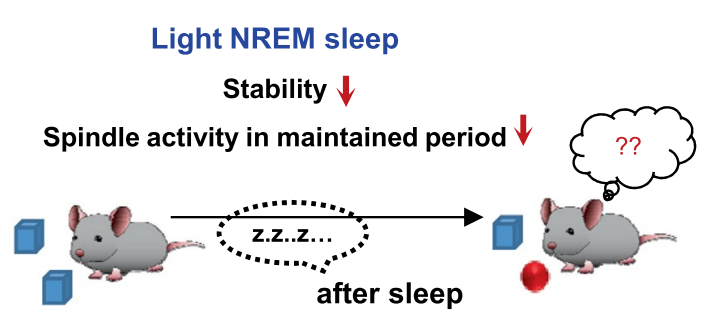

Figure 6. Differential correlation of light and deep NREM sleep components with memory consolidation. (a) The correlation between the episodic duration of the light NREM sleep and the discrimination index (DI) for a novel object. (b) The correlation between the episodic duration of the deep NREM sleep and the DI for novel object. (c) The correlation between the number of sleep spindle (SS) per epoch occurring in the entire light NREM sleep and the DI for novel object. (d) The correlation between the normalized $\delta$ power in the deep NREM sleep and the DI for novel object. (e) The correlation between the number of SS per epoch occurring in the maintained light NREM period and the DI for novel object. (f) The correlation between the number of SS per epoch occurring in the maintained deep NREM period and the DI for novel object. Each point represents a value obtained from one mouse $(\mathrm{n}=18$; control, black circle; PLC $\beta 4$ TC KO, red triangle). (g) A scheme summarizing the importance of light NREM sleep for declarative memory consolidation via its stability and spindle activities.

\section{Discussion}

Both NREM and REM sleep have been reported to positively contribute to memory function ${ }^{34}$. However, most studies have highlighted the importance of deep NREM or REM sleep in memory formation, and the role of light NREM sleep dynamics is not clear ${ }^{17}$. In the present study using TC-specific PLC $\beta 4 \mathrm{KO}$ mice, we demonstrate that opposite changes of composition in light and deep NREM sleep and their effect on memory consolidation.

NREM sleep accompanied large-amplitude oscillatory EEG patterns, which presumably reflect the synchronized oscillations in thalamocortical circuit, although the initiation of NREM has been mostly attributed to the preoptic area of the hypothalamus ${ }^{35}$. Both sleep spindles and $\delta$ waves, which predominantly occurred in light and deep NREM sleep, respectively, are known to be generated in thalamocortical circuit accompanying burst firing in TC neurons ${ }^{19,20}$. We previously reported that deletion of PLC $\beta 4$ dramatically increased burst firing in TC neurons ${ }^{26}$. We therefore investigated whether TC-specific PLC 34 KO would affect NREM sleep dynamics and NREM sleep rhythms. TC-specific PLC $\beta 4 \mathrm{KO}$ increased the total duration of NREM sleep, but not REM sleep compared to whole PLC $\beta 4 \mathrm{KO}$ which showed the changes in many aspects of sleep components probably due to the expression of PLC $\beta 4$ in other brain regions including brain stem and medial septum ${ }^{36}$. Especially, PLC $\beta 4$ TC KO mice displayed a significant increase in the duration of deep NREM sleep that occurred via enhancement of its occurrence and stability, which led to an increase in the total sleep duration. However, the duration of the light NREM sleep was reduced, with a weakened stability and no change in its occurrence (Fig. 3d). In brain rhythms, the spindle incidence in the light NREM state was reduced (Fig. 4d), although burst firing increased in PLC 34 TC KO mice ${ }^{26}$. This result supports previous reports that suggest that TC burst firing is not essential for spindle generation $^{33}$. Interestingly, the change of spindle incidence per epoch by PLC $\beta 4$ TC KO was observed only in light NREM state, but not deep NREM state (Fig. 4d and Supplementary Fig. S2b). This supports a previous proposal that fast spindles, which are most pronounced in light NREM sleep, are generated in thalamic nuclei, whereas slow spindles, which are most evident in deep NREM sleep, originate from the intracortical area, as well as thalamic region ${ }^{37,38}$. 
Many studies have suggested that an increase in NREM sleep duration facilitates declarative memory processing $^{39}$. However, we found that sleep-dependent declarative memory consolidation was impaired, despite the increase in NREM sleep duration (Fig. 5b). We observed a strong and positive correlation between declarative memory consolidation and the episodic duration of light NREM sleep in all tested animals together (Fig. 6a). When separating the control and PLC 34 TC KO groups, their positive correlation was observed only in the control group, suggesting that the stability of light NREM and memory consolidation has correlational nature. The correlation disappeared in PLC $\beta 4$ TC KO group where the light NREM stability was decreased (Supplementary Fig. S4a). A previous human study reported that the duration of light NREM sleep was positively correlated with procedural memory function ${ }^{40}$. Our results indicate that the stability of light NREM sleep has beneficial role in declarative memory functions. On the other hand, we found a negative correlation between total amount of deep NREM sleep and declarative memory consolidation, which conflicts with the results of several human studies that have reported deep NREM sleep to enhance the memory formation ${ }^{16,41}$. However, most of these previous studies could not exclude the effect involving enhancement of light NREM sleep for memory improvement because of limitations in their experimental method. Therefore, our results suggest that the abnormal increase in deep NREM sleep accompanied by a loss of light NREM sleep may impair the memory formation.

There have been diverse opinions about the role of $\delta$ waves, which are nested within slow sleep oscillations, in memory formation. The $\delta$ waves were considered to promote the performance of various types of memory $\mathrm{y}^{42}$. However, in patients with schizophrenia, $\delta$ power during deep NREM sleep is not associated with declarative memory function ${ }^{43}$. In the present study, we did not observe any correlation between the $\delta$ power in deep NREM state and declarative memory consolidation, regardless of PLCB4 deletion (Fig. 6d, Supplementary Fig. S4e).

Our data demonstrated that the spindle activity in the light NREM state had a positive correlation with declarative memory consolidation in all tested animals together (Fig. 6c). Their positive correlation was conserved in the control group, suggesting the correlational nature between the spindle activity of light NREM state and memory consolidation (Supplementary Fig. S4b). This supports that the decreased spindle activity of light NREM state would be linked to the sleep-dependent memory impairment observed in PLC $\beta 4$ TC KO mice. Despite the fact that spindles are observed throughout NREM sleep, many researchers, suggesting the importance of spindles for memory function, have highlighted light NREM sleep, where the spindles frequently occur ${ }^{44}$. It is unknown whether spindle activity that occurs during light vs. deep NREM or the maintained vs. transition period of NREM has a distinct role in memory function. We found a strong correlation with memory consolidation was observed when spindle occurred during the maintained light NREM period only and not during deep NREM or the transition period (Fig. 6e,f; Supplementary Fig. S3c). These results were confirmed when the control group was analyzed separately (Supplementary Fig. S4c and S4f). These results indicate that the positive role of spindles on memory formation depends on when the spindle occurs.

In summary, we demonstrated that declarative memory consolidation was positively correlated with the stability of light NREM sleep, irrespective of the total amount of NREM sleep. Moreover, this is the first study to report that the timing of spindle occurrence in light NREM state may be critical for the beneficial effect of spindles on memory consolidation. These findings highlight the importance of light NREM sleep for memory formation, and provide novel insights into a balance between light and deep NREM sleep, not simple sleep induction, as therapeutic and diagnostic targets for the treatment of sleep-related cognitive deficits.

\section{Methods}

Study animals. All experiments used Plc $\beta 4$ floxed mice from a C57BL/6J genetic background. The mice were maintained with free access to food and water under a 12-h light and 12-h dark cycle, with the light cycle beginning at 7:00 a.m. Animal care and handling were conducted in accordance with the guidelines of the Institutional Animal Care and Use Committee (IACUC) at Yonsei University (Seoul, Korea) and all experimental protocols were apporoved by the IACUC at Yonsei University (Approval ID number IACUC-A-201601-407-02).

Surgery. Twelve- to 14-week-old male mice were used for the chronic monitoring of the EEG/EMG signals. The mice were anesthetized with $0.2 \%$ tribromoethanol $(20 \mathrm{ml} / \mathrm{kg}$, intraperitoneal injection; Sigma-Aldrich, St. Louis, MO, USA) and placed on a stereotaxic frame (David Kopf Instruments, Tujunga, CA, USA). Cre-inducible vectors containing adeno-associated virus (AAV9.hsyn.HI.eGFP-Cre.WPRE.SV40) were bilaterally injected into the ventrobasal complex region of the thalamus (anteroposterior, $-1.82 \mathrm{~mm}$; lateral, $\pm 1.75 \mathrm{~mm}$; ventral, -3.5 and $-3.0 \mathrm{~mm}, 1 \mu \mathrm{l}$ in each site) using a NanoFil needle (NF33BL; World Precision Instruments, Sarasota, FL, USA) with a Hamilton syringe and pump (SP100IZ; World Precision Instruments, USA). AAV9.hsyn.eGFP.WPRE.bGH was used as control. For the EEG recordings, epidural electrodes with a 6-pin surface mount connector with EMG leads (8231-SM; Pinnacle Technology, USA) were implanted in the frontal and parietal lobes. For the EMG signal recordings, EMG leads were inserted into the nuchal musculature and a grounding electrode was implanted in the occipital region of the skull.

Chronic EEG/EMG monitoring. After a 2-week recovery, mice were placed in unrestrained chronic recording environments under 12-h light and 12-h dark conditions. There was a 1-week adaptation period to the recording systems. EEG and EMG signals were chronically acquired for $24 \mathrm{~h}$ using a three-channel biopotential recording system and Sirenia software (Pinnacle Technology, USA, RRID:SCR_016183). The signals were collected using a preamplifier (8202 mouse preamplifier for a 3-channel system; Pinnacle Technology, USA) with a low-pass filter at $400 \mathrm{~Hz}$ for EEG and high-pass filter at $10 \mathrm{~Hz}$ for EMG, and digitized at a sampling rate of $400 \mathrm{~Hz}$ (8206, EEG/EMG data conditioning and acquisition system; Pinnacle Technology, USA).

Novel object recognition procedures and behavioral analyses. On day 0 , an open field test was performed for $60 \mathrm{~min}$ in a quadratic recording box $(40 \times 40 \times 40 \mathrm{~cm})$ made of acrylic white polyvinyl chloride, 
which was the habituation session for the novel object recognition task; continuous video tracking was implemented to identify and exclude mice with abnormal locomotion. On the following day, the test was run on 2 consecutive days during the first $2 \mathrm{~h}$ of the light cycle onset (7:00 am to 9:00 am). On day 1, the mice were placed in a quadratic recording box and the same two identical objects (object 1) were each placed in a randomly assigned quadrant within the test area. The mice were allowed to explore freely for $10 \mathrm{~min}$ before being returned to their sleep recording chamber. On day 2 , one of the objects was randomly replaced by a new one (object 2 ). The mice were given $10 \mathrm{~min}$ to explore the test area. Test sessions on each day were recorded with an overhead video camera and analyzed using Ethovision XT (Noldus, Leesburg, VA, USA, RRID: SCR_000441). For the analysis of novel object preferences, the time spent exploring each object during the test period was measured for each mouse and preference was determined using the following equation: Discrimination index (DI) $=$ (object 2 exploration (sec) - object 1 exploration (sec)/(object 1 exploration (sec) + object 2 exploration (sec)). On day 1 , the novel object randomly indicated one of two objects and mice with side preference was excluded in data set (control, $\mathrm{n}=2$; PLC34 TC KO, $\mathrm{n}=2$ ).

After the experiment, all mice were placed in a Y-maze composed of three equally spaced arms $\left(120^{\circ} ; 30 \mathrm{~cm}\right.$ long). The arms were labeled by different patterns of masking tape to distinguish each arm and defined as A, B, and $\mathrm{C}$. In the training session, mice were allowed to move freely in two compartments (A and B) for 5 min before being returned to their home cage. After a 30-min break, the mice were again given $5 \mathrm{~min}$ to explore the test area, in which all three compartments (A, B, and C) were open. Short-term memory was indicated by the preference for a new arm and was determined using the following equation: new arm preference $(\%)=$ exploration time in $\mathrm{C}(\mathrm{sec}) /$ exploration time in $\mathrm{A}+\mathrm{B}(\mathrm{sec}) \times 100$.

Fear conditioning procedures and behavioral analyses. All mice were tested for context- and cue-based responses following a standard fear conditioning protocol. The tests were implemented within the first $2 \mathrm{~h}$ of the onset of the light cycle (7:00 a.m. to 9:00 a.m.). During the conditioning phase, mice were placed in a metallic rectangular chamber with a surface grid (context A) connected to an electrical shocker (Coulbourn Instruments, Hollistan, MA, USA). The mice were habituated to the box for $4 \mathrm{~min}$, followed by application of three tone-shock pairs separated by an interval of $2 \mathrm{~min}$. Each tone $(2 \mathrm{kHz}$ at $80 \mathrm{~dB})$ lasted for $28 \mathrm{sec}$ and was accompanied by a foot shock $(2 \mathrm{sec}, 0.5 \mathrm{~mA})$. Once conditioning was completed, the mice were returned to their sleep recording chamber. Between each conditioning session, the test area was cleaned with $70 \%$ ethanol and water.

On the following day, contextual and cued memory recall tests were completed $1 \mathrm{~h}$ apart within the first $2 \mathrm{~h}$ of the onset of the light cycle. For the context test, the mice were placed in the same chamber, as they had been for context A during conditioning, for $5 \mathrm{~min}$; however, no tone-shock events were delivered. Contextual fear memory was determined by the percentage of freezing time for $5 \mathrm{~min}$. Upon test completion, the mice were immediately returned to their sleep recording chamber for $\sim 1 \mathrm{~h}$. The test area was thoroughly cleaned with $70 \%$ ethanol and water between subsequent test sessions.

For cue testing, the test area (context B) was substantially different than that used for the conditioning session and context testing. The floor and walls were white Plexiglass panels, as opposed to the black walls of the testing area used for conditioning and context tests. Peroxyguard was used to clean the test area between mice. During the cue test, three $80 \mathrm{~dB}$ tones of $28 \mathrm{sec}$ separated by an interval of $2 \mathrm{~min}$, identical to those produced during conditioning, were presented. Cue fear to the tone was determined by the percentage of freezing time during a 3-min period ( 3 tone $\times 1 \mathrm{~min}$ following tone onset). For both the contextual and cue fear tests, freezing behavior was assessed by a blinded experimenter using behavior counter software, and freezing durations in the conditioning phase were presented across 10 -sec bins.

Sleep scoring and analysis. The parietal EEG/EMG recordings were scored semi-automatically using the SleepSign software sleep scoring system (Kissei Comtec, USA) in 8-sec epochs as awake (low voltage, high frequency EEG activity with a high amplitude EMG), NREM sleep (high amplitude, slow EEG activity with a reduction of the EMG tone), and REM sleep (low amplitude EEG comprised mainly of $\theta$-wave activity with EMG atonia). NREM sleep was further subdivided into the light and deep NREM by the ratio of the $\delta$ frequency $(0.5-4 \mathrm{~Hz})$ power $^{27-30}$. In each epoch, the total EEG power within the $0-20 \mathrm{~Hz}$ frequency range was normalized to $100 \%$. When the percentage of $\delta$ frequency power to total EEG power was less than $40 \%$ in one epoch, it was scored to the light NREM sleep. Deep NREM sleep was scored when the percentage of $\delta$ frequency power to total EEG power was higher than $40 \%$ in one epoch. The onset of sleep and wake episodes was defined as three consecutive epochs. Epochs containing artifacts that occurred during active wakefulness (with large movements) or containing two vigilance states were visually identified. Transition probability (from A to B state) was determined using the following equation: transition number to $\mathrm{B} /$ total number of transitions from $\mathrm{A} \times 100$.

Power spectral density analysis. To analyze the power spectral densities, the EEG spectral power in each 8 -sec epoch was calculated in $0.39-\mathrm{Hz}$ bins using fast Fourier transformation (Hamming window) and normalized within each sleep state using SleepSign software. The power bins in the $0-20 \mathrm{~Hz}$ range were summed for the three frequency bands $[\delta(0.5-4 \mathrm{~Hz}), \theta(4-9 \mathrm{~Hz}), \sigma(10-15 \mathrm{~Hz})]$ and then averaged within each group.

The spindle detection method. The incidence of sleep spindles during NREM sleep was analyzed using MATLAB software (MathWorks, RRID: SCR_001622). EEG traces of the light NREM state were obtained by dividing into period to be maintained or before transition to deep NREM or REM sleep. EEG traces of the deep NREM state were obtained in maintained periods. The transition period was defined as the five epochs before transition to other states or immediately after the transition. The epochs, except for those comprising the transition period, were selected as maintained periods. The EEG signal was bandpass filtered at $7-15 \mathrm{~Hz}$ and the 
envelope was determined using the Hilbert transform. A potential spindle event was identified in cases where the envelope exceeded a threshold, which was defined as the standard deviation of the baseline envelope multiplied by 2 . The spindles by identifying the center of detected spindles was presented as the number of spindle occurring in one epoch (8s) and averaged first for each mouse and then within groups. Subsequently, potential spindle events with durations shorter than $0.2 \mathrm{sec}$ or longer than $3 \mathrm{sec}$ were discarded.

Immunohistochemistry. For histological analysis, mice were anesthetized with $0.2 \%$ tribromoethanol $(20 \mathrm{ml} / \mathrm{kg}$, intraperitoneal injection) and transcardially perfused with $1 \mathrm{M}$ phosphate-buffered saline (PBS) followed by a $4 \%$ paraformaldehyde solution. After perfusions, the brains were fixed in $4 \%$ paraformaldehyde overnight and then submerged in $30 \%$ sucrose solution for 3 days at $4{ }^{\circ} \mathrm{C}$. The brains frozen in O.C.T. compound were cut into serial $40-\mu \mathrm{m}$ thick coronal sections on a freezing microtome, and stored in PBS. The brain sections were permeabilized with $0.1 \%$ Tween-20 in PBS for $30 \mathrm{~min}$ and then incubated in blocking solution (5\% normal goat serum in PBS) for $1 \mathrm{~h}$. After washing three times with PBS, the tissues were incubated with a primary antibody against PLC 34 (H-300; Santa Cruz Biotechnology, Santa Cruz, CA, USA; 1:200) for $24 \mathrm{~h}$ at $4{ }^{\circ} \mathrm{C}$. The tissues were rinsed three times in PBS, incubated with a Cy3-conjugated secondary antibody (Jackson ImmunoResearch Labs, West Grove, PA, USA, RRID: AB_2338000; 1:500) for $2 \mathrm{~h}$ at room temperature, and then mounted on microscope slides with fluorescent mounting media (Dako, Glostrup, Denmark). Fluorescence images were obtained with a LSM 880 confocal microscope (Carl Zeiss, Oberkochen, Germany). The image was acquired in consistent parameters across the conditions and selected the middle of the TC region by the experimenter blinded to the conditions. The cell counting was performed manually from the two other blinded experimenters.

Quantitative reverse transcription-PCR (qRT-PCR). Total RNA was isolated from the thalamus of $P l c \beta 4$ floxed mice injected with AAV.eGFP or AAV.eGFP-Cre, using TRIzol reagent (Invitrogen, Carlsbad, CA, USA). One $\mu$ g of total RNA was used for cDNA synthesis with the SuperScript III First-Strand Synthesis System for RT-PCR (Invitrogen, Carlsbad, CA, USA). qRT-PCR was performed using the CFX Connect Real-Time PCR Detection System (Bio-Rad, Hercules, CA, USA). The qRT-PCR primers used to assess expression were as follows: $\beta$-actin: F1 primer $5^{\prime}$-TGTGATGGTGGGAATGGGTCAGAA- $3^{\prime}$ and R1 primer $5^{\prime}$-TGTGGTGCCAGATCTTCTCCATGT-3' to produce a $140 \mathrm{bp}$ cDNA; $P l c \beta 1$ : F2 primer $5^{\prime}$-TCGTACATCCAGGAGGTGGT-3' and R2 primer $5^{\prime}$-CTGCAGCTTGGGCTTCTCAT- $3^{\prime}$ to produce a $129 \mathrm{bp}$ cDNA; Plc $\beta 4$ primer 1: F3 primer $5^{\prime}$-CTGGAAGGGCGGATATTGTGT-3' and R3 primer $5^{\prime}$-ATCGGACTGACGTTGTTTGC- $3^{\prime}$ to produce a $155 \mathrm{bp}$ cDNA; $P l c \beta 4$ primer 2: F 4 primer $5^{\prime}$-GGGCGGATATTGTGTGTCTG-3' and R4 primer 5'-TGTTGGTCAGAAAGGCCAGTT-3' to produce a 196 bp cDNA. Gene expression was quantified using the comparative CT method with $\beta$-actin as the reference gene.

In vitro intra-thalamic oscillation recordings. For intra-thalamic oscillation recordings, mice were anesthetized by halothane and decapitated. The brains were sectioned to achieve $400 \mu \mathrm{m}$-thick horizontal slices using VT1000s (Leica Microsystems, Wetzlar, Germany). The slices were stabilized for at least $1 \mathrm{~h}$ in artificial cerebrospinal fluid (aCSF) containing $\mathrm{NaCl}, 130 \mathrm{mM} ; \mathrm{NaHCO}_{3}, 24 \mathrm{mM} ; \mathrm{KCl}, 3.5 \mathrm{mM} ; \mathrm{NaH}_{2} \mathrm{PO}_{4}, 1.25 \mathrm{mM} ; \mathrm{CaCl}_{2}$, $1.5 \mathrm{mM} ; \mathrm{MgCl}_{2}, 0.75 \mathrm{mM}$; and glucose, $10 \mathrm{mM}$, and simultaneously equilibrated with $95 \% \mathrm{O}_{2} / 5 \% \mathrm{CO}_{2}$ at $25^{\circ} \mathrm{C}$. After stabilization, intra-thalamic oscillations were evoked by a $300 \mu \mathrm{A}$ and $100 \mu$ square pulse stimulus in the internal capsule (IC) through a bipolar tungsten electrode (FHC, Bowdoin, ME, USA). The stimulus interval was $30 \mathrm{sec}$. Extracellular potentials evoked by IC stimulation were measured in ventro-posterolateral nuclei of thalamus using a tungsten electrode $(\sim 100 \mathrm{k} \Omega$ ). Signals were amplified using MultiClamp 700B (Molecular Devices, San Jose, CA, USA, RRID:SCR_011323), and data acquisition was performed using a Digitizer 1440 A and Clampex (Molecular Devices). The measured signals were bandpass filtered at $10 \mathrm{~Hz}-2 \mathrm{kHz}$.

In vitro whole-cell patch-clamp recordings. The burst firings were recorded under aCSF solution $(\mathrm{NaCl}$, $124 \mathrm{mM}$; KCl, 3 mM; $\mathrm{MgSO}_{4}, 1.3 \mathrm{mM} ; \mathrm{NaH}_{2} \mathrm{PO}_{4}, 1.25 \mathrm{mM} ; \mathrm{NaHCO}_{3}, 26 \mathrm{mM} ; \mathrm{CaCl}_{2}-2 \mathrm{H}_{2} \mathrm{O}, 2.4 \mathrm{mM}$; and glucose, $10 \mathrm{mM}$ ) and aerated with $95 \% \mathrm{O}_{2} / 5 \% \mathrm{CO}_{2}$ by whole-cell current-clamp. Patch electrodes (4-7M $\Omega$ ) fabricated from standard-wall borosilicate glass (GC150F-10, Warner Instrument Corp., USA) were filled with an intra-pipette solution containing K-gluconate, $145 \mathrm{mM}$; HEPES, $10 \mathrm{mM}$; NaCl, $5 \mathrm{mM}$; EGTA, $0.2 \mathrm{mM}$; Mg-ATP, $5 \mathrm{mM}$; and $\mathrm{Na}_{2}-\mathrm{GTP}, 0.5 \mathrm{mM}$, with $\mathrm{pH}$ adjusted to 7.3 and osmolality adjusted to $285 \sim 295 \mathrm{mOsmol} / \mathrm{kg}$. Rebound T-type calcium channel-mediated burst firing was measured after one-second-hyperpolarizing current step. The number of intra-burst spike was identified in cases where the amplitude of action potential exceeded a threshold, which was defined as "dV/dt $(\mathrm{mV} / \mathrm{ms})>10 \mathrm{mV} / \mathrm{ms}^{\text {" }} 45,46$.

Statistical analysis. All data were initially confirmed that is well-modeled by a normal using D'Agostino-Pearson normality test. Between-group differences were analyzed using unpaired $t$-tests with Welch's correction. Data are presented as the mean \pm standard error of the mean (SEM) and a value of $\mathrm{p}<0.05$ was considered statistically significant. Linear correlation was assessed by simple linear regression analysis using GraphPad Prism 8.3.1 (GraphPad Software, San Diego, CA, USA).

\section{Data availability}

The datasets generated during and/or analyzed during the current study are available from the corresponding author on reasonable request.

Received: 3 October 2019; Accepted: 15 April 2020;

Published online: 01 June 2020 


\section{References}

1. Siegel, J. M. Sleep viewed as a state of adaptive inactivity. Nat Rev Neurosci 10, 747-753, https://doi.org/10.1038/nrn2697 (2009).

2. Xie, L. et al. Sleep drives metabolite clearance from the adult brain. Science 342, 373-377, https://doi.org/10.1126/science.1241224 (2013).

3. Schmidt, M. H. The energy allocation function of sleep: a unifying theory of sleep, torpor, and continuous wakefulness. Neurosci Biobehav Rev 47, 122-153, https://doi.org/10.1016/j.neubiorev.2014.08.001 (2014).

4. Tononi, G. \& Cirelli, C. Sleep function and synaptic homeostasis. Sleep Med Rev 10, 49-62, https://doi.org/10.1016/j. smrv.2005.05.002 (2006).

5. Walker, M. P. The role of sleep in cognition and emotion. Ann N Y Acad Sci 1156, 168-197, https://doi.org/10.1111/j.17496632.2009.04416.x (2009).

6. Rasch, B. \& Born, J. About sleep's role in memory. Physiol Rev 93, 681-766, https://doi.org/10.1152/physrev.00032.2012 (2013).

7. Korman, M. et al. Daytime sleep condenses the time course of motor memory consolidation. Nat Neurosci 10, 1206-1213, https:// doi.org/10.1038/nn1959 (2007).

8. Rolls, A. et al. Optogenetic disruption of sleep continuity impairs memory consolidation. Proc Natl Acad Sci USA 108, 13305-13310, https://doi.org/10.1073/pnas.1015633108 (2011)

9. Graves, L. A., Heller, E. A., Pack, A. I. \& Abel, T. Sleep deprivation selectively impairs memory consolidation for contextual fear conditioning. Learn Mem 10, 168-176, https://doi.org/10.1101/lm.48803 (2003).

10. Dresler, M., Kluge, M., Genzel, L., Schussler, P. \& Steiger, A. Impaired off-line memory consolidation in depression. Eur Neuropsychopharmacol 20, 553-561, https://doi.org/10.1016/j.euroneuro.2010.02.002 (2010).

11. Wamsley, E. J. et al. Reduced sleep spindles and spindle coherence in schizophrenia: mechanisms of impaired memory consolidation? Biol Psychiatry 71, 154-161, https://doi.org/10.1016/j.biopsych.2011.08.008 (2012).

12. Seeck-Hirschner, M. et al. Effects of daytime naps on procedural and declarative memory in patients with schizophrenia. JPsychiatr Res 44, 42-47, https://doi.org/10.1016/j.jpsychires.2009.05.008 (2010).

13. Rechtschaffen A K. A. A manual of standardized terminology, techniques and scoring system for sleep stages of human subjects. (Los Angeles, CA: Brain Information Service/Brain Research Institute, University of California, 1968).

14. Ackermann, S. \& Rasch, B. Differential effects of non-REM and REM sleep on memory consolidation? Curr Neurol Neurosci Rep 14, 430, https://doi.org/10.1007/s11910-013-0430-8 (2014).

15. Plihal, W. \& Born, J. Effects of early and late nocturnal sleep on declarative and procedural memory. J Cogn Neurosci 9, 534-547, https://doi.org/10.1162/jocn.1997.9.4.534 (1997).

16. Tucker, M. A. et al. A daytime nap containing solely non-REM sleep enhances declarative but not procedural memory. Neurobiol Learn Mem 86, 241-247, https://doi.org/10.1016/j.nlm.2006.03.005 (2006).

17. Genzel, L., Kroes, M. C., Dresler, M. \& Battaglia, F. P. Light sleep versus slow wave sleep in memory consolidation: a question of global versus local processes? Trends Neurosci 37, 10-19, https://doi.org/10.1016/j.tins.2013.10.002 (2014).

18. Steriade, M., McCormick, D. A. \& Sejnowski, T. J. Thalamocortical oscillations in the sleeping and aroused brain. Science 262, 679-685 (1993).

19. Contreras, D. \& Steriade, M. Spindle oscillation in cats: the role of corticothalamic feedback in a thalamically generated rhythm. $J$ Physiol 490(Pt 1), 159-179 (1996).

20. von Krosigk, M., Bal, T. \& McCormick, D. A. Cellular mechanisms of a synchronized oscillation in the thalamus. Science 261, 361-364 (1993).

21. Crandall, S. R., Cruikshank, S. J. \& Connors, B. W. A corticothalamic switch: controlling the thalamus with dynamic synapses. Neuron 86, 768-782, https://doi.org/10.1016/j.neuron.2015.03.040 (2015).

22. McCormick, D. A. \& von Krosigk, M. Corticothalamic activation modulates thalamic firing through glutamate "metabotropic" receptors. Proc Natl Acad Sci U S A 89, 2774-2778 (1992).

23. Vidnyanszky, Z. et al. Immunocytochemical visualization of the mGluRla metabotropic glutamate receptor at synapses of corticothalamic terminals originating from area 17 of the rat. Eur J Neurosci 8, 1061-1071 (1996).

24. Miyata, M. et al. Role of thalamic phospholipase C[beta] 4 mediated by metabotropic glutamate receptor type 1 in inflammatory pain. J Neurosci 23, 8098-8108 (2003).

25. Cheong, E. et al. Tuning thalamic firing modes via simultaneous modulation of T- and L-type Ca2+ channels controls pain sensory gating in the thalamus. J Neurosci 28, 13331-13340, https://doi.org/10.1523/JNEUROSCI.3013-08.2008 (2008).

26. Cheong, E. et al. Deletion of phospholipase C beta4 in thalamocortical relay nucleus leads to absence seizures. Proc Natl Acad Sci U S A 106, 21912-21917, https://doi.org/10.1073/pnas.0912204106 (2009).

27. Münch, A. D. M., Hescheler, J. \& Schneider, T. CaV2.3 E-/R-type voltage-gated calcium channels modulate sleep in mice. Somnologie 3, 185-192, https://doi.org/10.1007/s11818-013-0628-7 (2013).

28. Schneider, T. \& Dibue-Adjei, M. Ca 2.3 E-/R-type voltage-gated calcium channels modulate sleep in mice. Sleep 38, 499, https://doi. org/10.5665/sleep.4518 (2015).

29. Neckelmann, D. \& Ursin, R. Sleep stages and EEG power spectrum in relation to acoustical stimulus arousal threshold in the rat. Sleep 16, 467-477 (1993).

30. Popa, D., El Yacoubi, M., Vaugeois, J. M., Hamon, M. \& Adrien, J. Homeostatic regulation of sleep in a genetic model of depression in the mouse: effects of muscarinic and 5-HT1A receptor activation. Neuropsychopharmacology 31, 1637-1646, https://doi. org/10.1038/sj.npp.1300948 (2006).

31. Kishi, A., Struzik, Z. R., Natelson, B. H., Togo, F. \& Yamamoto, Y. Dynamics of sleep stage transitions in healthy humans and patients with chronic fatigue syndrome. Am J Physiol Regul Integr Comp Physiol 294, R1980-1987, https://doi.org/10.1152/ ajpregu.00925.2007 (2008).

32. Vyazovskiy, V. V., Achermann, P., Borbely, A. A. \& Tobler, I. The dynamics of spindles and EEG slow-wave activity in NREM sleep in mice. Arch Ital Biol 142, 511-523 (2004).

33. Lee, J. et al. Sleep spindles are generated in the absence of T-type calcium channel-mediated low-threshold burst firing of thalamocortical neurons. Proc Natl Acad Sci U S A 110, 20266-20271, https://doi.org/10.1073/pnas.1320572110 (2013).

34. Rauchs, G., Desgranges, B., Foret, J. \& Eustache, F. The relationships between memory systems and sleep stages. J Sleep Res 14, 123-c40, https://doi.org/10.1111/j.1365-2869.2005.00450.x (2005).

35. Chen, K. S. et al. A Hypothalamic Switch for REM and Non-REM Sleep. Neuron 97, 1168-1176 e1164, https://doi.org/10.1016/j. neuron.2018.02.005 (2018).

36. Hong, J. et al. The thalamic mGluR1-PLCbeta4 pathway is critical in sleep architecture. Mol Brain 9, 100, https://doi.org/10.1186/ s13041-016-0276-5 (2016).

37. Ayoub, A. et al. Differential effects on fast and slow spindle activity, and the sleep slow oscillation in humans with carbamazepine and flunarizine to antagonize voltage-dependent $\mathrm{Na}^{+}$and $\mathrm{Ca}^{2+}$ channel activity. Sleep 36, 905-911, https://doi.org/10.5665/ sleep.2722 (2013).

38. Timofeev, I. \& Chauvette, S. The spindles: are they still thalamic? Sleep 36, 825-826, https://doi.org/10.5665/sleep.2702 (2013).

39. Gais, S. \& Born, J. Declarative memory consolidation: mechanisms acting during human sleep. Learn Mem 11, 679-685, https://doi. org/10.1101/lm.80504 (2004).

40. Walker, M. P., Brakefield, T., Morgan, A., Hobson, J. A. \& Stickgold, R. Practice with sleep makes perfect: sleep-dependent motor skill learning. Neuron 35, 205-211 (2002). 
41. Walker, M. P. The role of slow wave sleep in memory processing. J Clin Sleep Med 5, S20-26 (2009).

42. Marshall, L., Helgadottir, H., Molle, M. \& Born, J. Boosting slow oscillations during sleep potentiates memory. Nature 444, 610-613, https://doi.org/10.1038/nature05278 (2006).

43. Goder, R. et al. Delta power in sleep in relation to neuropsychological performance in healthy subjects and schizophrenia patients. J Neuropsychiatry Clin Neurosci 18, 529-535, https://doi.org/10.1176/jnp.2006.18.4.529 (2006).

44. Schabus, M. et al. Sleep spindles and their significance for declarative memory consolidation. Sleep 27, 1479-1485 (2004)

45. Yang, Z. \& Santamaria, F. Purkinje cell intrinsic excitability increases after synaptic long term depression. J Neurophysiol 116, 1208-1217, https://doi.org/10.1152/jn.00369.2016 (2016).

46. Kress, G. J., Dowling, M. J., Meeks, J. P. \& Mennerick, S. High threshold, proximal initiation, and slow conduction velocity of action potentials in dentate granule neuron mossy fibers. J Neurophysiol 100, 281-291, https://doi.org/10.1152/jn.90295.2008 (2008).

\section{Acknowledgements}

Supported by the National Research Foundation of Korea grant (NRF) (2017R1A2B3011098, 2014M3A7B4051596, 2017M3C7A1023471), the Korea Health Technology R\&D Project through the Korea Health Industry Development Institute, and the Ministry of Health \& Welfare, Republic of Korea (HI18C1664) and the Brain Korea 21(BK21) PLUS program/ E.C., J.H., G.E.H., H.K., Y.L. and H.J. are fellowship awardee by BK21 PLUS program.

\section{Author contributions}

E.C. supervised the project. J.H., G.E.H., H.K., Y.L. and H.J. performed the experiments and analyzed the data. J.H. managed the direction of the study and conducted in vivo electrophysiology and behavioral experiments. G.E.H. conducted RT-PCR. H.K. conducted in vitro electrophysiology experiments. Y.L. and H.J. was involved in spindle analysis and immunohistochemistry assays. P.S. produced the mice for experiments. J.H. and E.C. interpreted the results and wrote the manuscript. All authors read and approved the final manuscript.

\section{Competing interests}

The authors declare no competing interests.

\section{Additional information}

Supplementary information is available for this paper at https://doi.org/10.1038/s41598-020-64377-7.

Correspondence and requests for materials should be addressed to E.C.

Reprints and permissions information is available at www.nature.com/reprints.

Publisher's note Springer Nature remains neutral with regard to jurisdictional claims in published maps and institutional affiliations.

(c) (i) Open Access This article is licensed under a Creative Commons Attribution 4.0 International License, which permits use, sharing, adaptation, distribution and reproduction in any medium or format, as long as you give appropriate credit to the original author(s) and the source, provide a link to the Creative Commons license, and indicate if changes were made. The images or other third party material in this article are included in the article's Creative Commons license, unless indicated otherwise in a credit line to the material. If material is not included in the article's Creative Commons license and your intended use is not permitted by statutory regulation or exceeds the permitted use, you will need to obtain permission directly from the copyright holder. To view a copy of this license, visit http://creativecommons.org/licenses/by/4.0/.

(C) The Author(s) 2020 